\title{
Impurity transport driven by ion temperature gradient turbulence in tokamak plasmas
}

\author{
T. Fülöp, ${ }^{1}$ S. Braun, ${ }^{2}$ and I. Pusztai ${ }^{1}$ \\ ${ }^{1}$ Department of Applied Physics, Nuclear Engineering, Chalmers University of Technology \\ and Euratom-VR Association, Göteborg SE-41296, Sweden \\ ${ }^{2}$ Max-Planck-Institut für Plasmaphysik, Greifswald 17491, Germany
}

(Received 4 March 2010; accepted 26 April 2010; published online 8 June 2010)

\begin{abstract}
Impurity transport driven by electrostatic turbulence is analyzed in weakly collisional tokamak plasmas using a semianalytical model based on a boundary layer solution of the gyrokinetic equation. Analytical expressions for the perturbed density responses are derived and used to determine the stability boundaries and the quasilinear particle fluxes. For moderate impurity charge number $Z$, the stability boundaries are very weakly affected by the increasing impurity charge for constant effective charge, while for lower impurity charge the influence of impurities is larger, if the amount of impurities is not too small. Scalings of the mode frequencies and quasilinear fluxes with charge number, effective charge, impurity density scale length, and collisionality are determined and compared to quasilinear gyrokinetic simulations with GYRO [J. Candy and R. E. Waltz, J. Comput. Phys. 186, 545 (2003)] resulting in very good agreement. Collisions do not affect the mode frequencies, growth rates, and impurity fluxes significantly. The eigenfrequencies and growth rates depend only weakly on $Z$ and $Z_{\text {eff }}$ but they are sensitive to the impurity density gradient scale length. An analytical approximate expression of the zero-flux impurity density gradient is derived and used to discuss its parametric dependencies. (C) 2010 American Institute of Physics.
\end{abstract}

[doi:10.1063/1.3430639]

\section{INTRODUCTION}

Understanding impurity transport in tokamak plasmas is important since fusion performance is significantly affected by impurities. In particular, impurity accumulation in the core may lead to fuel dilution and radiation power losses resulting in lower fusion power and potentially even plasma disruptions. However, impurities are welcome at the edge since they can prevent high heat fluxes to the wall by creating a radiative belt. Models of impurity transport driven by microturbulence ${ }^{1-15}$ and neoclassical processes ${ }^{16-18}$ are now well developed, but there are still many open issues regarding the sign and magnitude of the impurity particle flux and its parametric dependencies. To get reliable predictions for the turbulent fluxes, nonlinear electromagnetic gyrokinetic (GK) simulations are needed, but these are costly in computing time. However, the quasilinear electrostatic approximation has been proven to retain much of the relevant physics and reproduce the results of nonlinear GK simulations for a wide range of parameters. ${ }^{19}$ Reduced theoretical models, based on quasilinear approximations, benchmarked to GK simulations can ease the interpretation of the results of experiments or numerical simulations and can contribute to the better understanding of the underlying processes.

The aim of the present work is to calculate the quasilinear GYrokinetic IMpurity transport driven by ElectroStatic turbulence (GYIMES) using a semianalytical model based on a boundary layer solution of the GK equation. Following the approach of Refs. 20 and 21, we use a model electrostatic potential motivated by a variational analysis and GK simulations, and present analytical expressions for the perturbed density of the electrons, ions, and impurities. These are used in the quasineutrality equation that is solved numerically to obtain the frequencies and growth rates of the unstable modes, including the effect of impurities on these modes and the quasilinear impurity particle fluxes. In particular, we study the parametric dependences of the above quantities with respect to impurity charge, impurity density, inverse impurity scale length and collisionality, and the effect of the impurities on the stability boundaries. Using the analytically calculated expression for the perturbed impurity density response, we derive an approximative expression for the zeroflux impurity density gradient $R / L_{n z}$. Here $R$ is the major radius and $L_{n z}$ is the impurity density scale length. Such a zero impurity flux region is relevant to steady state plasmas in the core of tokamaks since the impurity influx occurs through the edge, and a simple analytical approximation for the zero-flux impurity density gradient is useful to be able to analyze its parametric dependencies. The results of GYIMES are compared to linear GK simulations with GYRO, ${ }^{22}$ and the agreement is very good.

The remainder of the paper is organized as follows. In Sec. II, the perturbed electron, ion, and impurity density and temperature responses are calculated. In Sec. III, the dispersion relation is presented and the dependence of the stability boundaries on the charge number and fraction of impurities is studied. In Sec. IV, the quasilinear transport fluxes are calculated and scalings of the growth rates, eigenfrequencies, and fluxes with charge number, fraction of impurities, impurity density scale length, and collisionality are discussed and compared to GYRO results. Finally, the results are summarized in Sec. V. 


\section{PERTURBED DENSITY RESPONSE}

The perturbed electron, ion, and impurity responses are obtained from the linearized GK equation, ${ }^{23}$

$$
\begin{gathered}
\frac{v_{\|}}{q R} \frac{\partial g_{a}}{\partial \theta}-i\left(\omega-\omega_{D a}\right) g_{a}-C_{a}\left(g_{a}\right) \\
\quad=-i \frac{e_{a} f_{a 0}}{T_{a}}\left(\omega-\omega_{* a}^{T}\right) \phi J_{0}\left(z_{a}\right),
\end{gathered}
$$

where $g_{a}$ is the nonadiabatic part of the perturbed distribution function, $\theta$ is the extended poloidal angle, ${ }^{24} \phi$ is the perturbed electrostatic potential, $f_{a 0}=n_{a} /\left(\sqrt{\pi} v_{T a}\right)^{3} \exp \left(-x_{a}^{2}\right)$ is the equilibrium Maxwellian distribution function, $x_{a}=v / v_{T a}$ is the velocity normalized to the thermal speed $v_{T a}$ $=\left(2 T_{a} / m_{a}\right)^{1 / 2}, n_{a}, T_{a}, m_{a}$, and $e_{a}$ are the density, temperature, mass, and charge of species $a$, respectively, $\omega_{* a}$ $=-k_{\theta} T_{a} / e_{a} B L_{n a}$ is the diamagnetic frequency, $\omega_{* a}^{T}=\omega_{* a}[1$ $\left.+\left(x_{a}^{2}-\frac{3}{2}\right) \eta_{a}\right], \quad \eta_{a}=L_{n a} / L_{T a}, L_{n a}=-\left[\partial\left(\ln n_{a}\right) / \partial r\right]^{-1}$, and $L_{T a}$ $=-\left[\partial\left(\ln T_{a}\right) / \partial r\right]^{-1}$ are the density and temperature scale lengths, $k_{\theta}$ is the poloidal wave number, $\omega_{D a}=-k_{\theta}\left(v_{\perp}^{2} / 2\right.$ $\left.+v_{\|}^{2}\right)(\cos \theta+s \theta \sin \theta) / \omega_{c a} R$ is the magnetic drift frequency, $\omega_{c a}=e_{a} B / m_{a}$ is the cyclotron frequency, $B$ is the equilibrium magnetic field, $q$ is the safety factor, $s=(r / q)(d q / d r)$ is the magnetic shear, $r$ and $R$ are the minor and major radii, and $J_{0}$ is the Bessel function of the first kind and $z_{a}=k_{\perp} v_{\perp} / \omega_{c a}$. We consider an axisymmetric, large aspect ratio torus with circular magnetic surfaces. We assume the following ordering of the electron/ion bounce frequencies and the eigenfrequency of the mode, $\omega_{b i} \ll \omega \ll \omega_{b e}$, and consider weakly collisional plasmas so that $\nu_{\star e}=\nu_{e} / \epsilon \omega_{b e} \ll 1$, where $\epsilon=r / R$ is the inverse aspect ratio and $\nu_{e}$ is the electron collision frequency, including collisions with ions and impurities, $\nu_{e}=\left(\hat{\nu}_{e e}+\hat{\nu}_{e i}+\hat{\nu}_{e z}\right) / x_{e}^{3}=\left(1+n_{i} / n_{e}+Z^{2} n_{z} / n_{e}\right) \hat{\nu}_{e e} / x_{e}^{3}$ $=\left(1+Z_{\mathrm{eff}}\right) \hat{\nu}_{e e} / x_{e}^{3}$, with $\hat{\nu}_{e e}=n_{e} e^{4} \ln \Lambda /\left(4 \pi \epsilon_{0}^{2} m_{e}^{2} v_{T e}^{3}\right)$, and $\ln \Lambda$ is the Coulomb logarithm. The electron collisions are modeled by a pitch-angle scattering operator

$$
C_{e}=\nu_{e}(v) \frac{2 \xi}{B} \frac{\partial}{\partial \lambda} \xi \lambda \frac{\partial}{\partial \lambda} \equiv \nu_{e}(v) \mathcal{L},
$$

where $\xi=v_{\|} / v$ and $\lambda=v_{\perp}^{2} /\left(B v^{2}\right)$. Collisions are neglected in the ion and impurity equations. The condition for which impurity collisions can be neglected is

$$
\left(Z^{3} m_{e} / m_{i}\right)^{1 / 2}\left(n_{z} Z^{2} / n_{i}\right) \epsilon \nu_{\star e} \ll 1
$$

and is well-satisfied for not too high charge numbers (depending on the exact parameters, up to $Z \approx 15-25$ ) if we assume, consistent with the rest of our orderings, that $\epsilon \ll 1$, $\nu_{\star e} \ll 1$, and $n_{z} Z^{2} / n_{i}=O(1)$. Note that the condition in Eq. (3) breaks down for very high charge numbers, specially when $\nu_{\star e}$ is not too small, in which case collisional effects can modify the impurity fluxes as shown in Ref. 14. As in Ref. 20, we use the following perturbed electrostatic potential:

$\phi(\theta)=\phi_{0}\left(\frac{1+\cos \theta}{2}+i f_{s} \sin ^{2} \theta\right)[H(\theta+\pi)-H(\theta-\pi)]$,

where $H$ is the Heaviside function and $f_{s}$ can approximately be written as $f_{s}=-0.6 s+s^{2}-0.3 s^{3}$. In Ref. 20, this model potential was motivated with a variational method and expe- rience from GYRO simulations. The approximation for the perturbed electrostatic potential breaks down for low and high shears (outside the region $0.2<s<1.7$ ) or near marginal instability, and correct quantitative predictions for the transport can only be expected in regions where the assumption for the potential is valid.

\section{A. Electron response}

The circulating electrons are assumed to be adiabatic and the nonadiabatic trapped electron distribution can be expanded $g_{e}=g_{e 0}+g_{e 1}+\cdots$ in the smallness of $\omega / \omega_{b e}$ and the normalized collisionality $\nu_{\star e}$, which gives $\partial g_{e 0} / \partial \theta=0$ in lowest order. The electron GK equation is orbit averaged between the mirror reflection points, providing a constraint for $g_{e 0}$,

$$
\begin{gathered}
\left(\omega-\left\langle\omega_{D e}\right\rangle\right) g_{e 0}-\frac{i \nu_{e}}{\epsilon K(\kappa)} \frac{\partial}{\partial \kappa} \hat{J}(\kappa) \frac{\partial g_{e 0}}{\partial \kappa} \\
=-\frac{e\langle\phi\rangle}{T_{e}}\left(\omega-\omega_{* e}^{T}\right) f_{e 0},
\end{gathered}
$$

where $\langle\cdots\rangle$ is an average over the bounce orbit of the trapped electrons, $\kappa=\left[1-\lambda B_{0}(1-\epsilon)\right] /\left(2 \epsilon \lambda B_{0}\right), B_{0}$ is the fluxsurface averaged magnetic field, $\hat{J}=E(\kappa)+(\kappa-1) K(\kappa)$, and $E(\kappa)$ and $K(\kappa)$ are the complete elliptic integrals. Here, the bounce average of the potential is

$$
\langle\phi\rangle=\phi_{0}\left\{\frac{E(\kappa)}{K(\kappa)}+i \frac{4 f_{s}}{3}\left[(2 \kappa-1) \frac{E(\kappa)}{K(\kappa)}+1-\kappa\right]\right\}
$$

and the orbit-averaged precession frequency for trapped electrons is

$$
\left\langle\omega_{D e}\right\rangle=\omega_{D 0}\left[\frac{E(\kappa)}{K(\kappa)}-\frac{1}{2}+2 s\left(\frac{E(\kappa)}{K(\kappa)}+\kappa-1\right)\right],
$$

with $\omega_{D 0}=-k_{\theta} v^{2} / \omega_{c e} R$. We introduce the parameter $\hat{\nu} \equiv \nu_{e} / \omega_{0} \epsilon$, which will be treated as small in the following analysis. This condition restricts the applicability of the solution for high temperature plasmas (such as ITER). Here, $\omega_{0}$ is the absolute value of the real part of the eigenfrequency so that $\omega=\sigma \omega_{0}+i \gamma=(\sigma+i \hat{\gamma}) \omega_{0} \equiv y \omega_{0}, \sigma=\operatorname{sign}(\Re\{\omega\})$ denotes the sign of the real part of the eigenfrequency, and $\hat{\gamma}=\gamma / \omega_{0}$ is the normalized growth rate.

In the limit of small collisionalities, we can construct a boundary layer solution to the GK equation

$$
\begin{aligned}
& \hat{\nu}\left[g_{e 0}^{\prime \prime}+(\ln \hat{J})^{\prime} g_{e 0}^{\prime}\right]+i \frac{K(\kappa)}{\hat{J} \omega_{0}}\left(\omega-\left\langle\omega_{D e}\right\rangle\right) g_{e 0} \\
& \quad=i \frac{K(\kappa) e\langle\phi\rangle}{\hat{J} \omega_{0} T_{e}}\left(\omega_{* e}^{T}-\omega\right) f_{e 0} .
\end{aligned}
$$

In the outer region, far away from the trapped/passing boundary, collisions can be neglected, and the solution to Eq. (7) is 


$$
g_{\text {outer }}=\frac{e\langle\phi\rangle\left(\omega_{* e}^{T}-\omega\right) f_{e 0}}{T_{e}\left(\omega-\left\langle\omega_{D e}\right\rangle\right)} .
$$

In the inner region, we can approximate $\hat{J}(\kappa)$ with its asymptotic limit for $\kappa \rightarrow 1: \hat{J}(\kappa) \simeq 1$. Furthermore we assume that in the inner region $g_{e 0}^{\prime \prime} \gg(\ln \hat{J})^{\prime} g_{e 0}^{\prime}$, the validity of which is checked a posteriori. Changing variables in Eq. (7) to $t$ $=(1-\kappa) / \sqrt{\hat{\nu}}$ gives

$$
\begin{gathered}
\frac{\partial^{2} g_{\text {inner }}}{\partial t^{2}}+i \frac{K(\kappa)}{\omega_{0}}\left(\omega-\left\langle\omega_{D e}\right\rangle\right) g_{\text {inner }} \\
=i \frac{e\langle\phi\rangle K(\kappa)\left(\omega_{* e}^{T}-\omega\right) f_{e 0}}{\omega_{0} T_{e}}
\end{gathered}
$$

and the solution is

$$
\begin{aligned}
g_{\text {inner }}= & g_{\text {outer }}+\hat{c}_{1} \exp [-(1-\kappa) \sqrt{\hat{u} K(\kappa) / \hat{\nu}}] \\
& +\hat{c}_{2} \exp [(1-\kappa) \sqrt{\hat{u} K(\kappa) / \hat{\nu}}]
\end{aligned}
$$

where $\hat{u}=-i\left(y-\left\langle\omega_{D e}\right\rangle / \omega_{0}\right) . \hat{c}_{1}$ is determined by the boundary condition $g_{e 0}(\kappa=1)=0$ and $\hat{c}_{2}=0$ to match the inner and outer solutions. The global solution is then

$$
g_{e 0}=g_{\text {outer }}(1-\exp [-(1-\kappa) \sqrt{\hat{u} K(\kappa) / \hat{\nu}}]),
$$

which agrees very well with the numerical solution of Eq. (5) for experimentally relevant parameters, as it will be shown in the Appendix. Interestingly, the boundary layer solution is a very good approximation of the numerically obtained distribution function $g_{e 0}$ even when $\hat{\nu}$ is of order unity.

Performing the velocity-space integration as outlined in the Appendix gives the following expression for the perturbed electron density response:

$$
\begin{aligned}
\frac{\hat{n}_{e}}{n_{e}} / \frac{e \phi}{T_{e}}= & -\widetilde{\phi}\left\{\sqrt { 2 \epsilon } \left[\hat{\omega}_{\eta^{* e}}\right.\right. \\
& \left.-\frac{3}{2}\left(\eta_{e} \widetilde{\omega}_{* e}-\frac{\widetilde{\omega}_{D t}}{2} \hat{\omega}_{\eta^{* e}}\right) \mathcal{F}_{5 / 2}^{1}\left(\frac{\widetilde{\omega}_{D t}}{2}\right)\right] \\
& -\frac{\Gamma(3 / 4) \sqrt{\epsilon \hat{\nu}_{t}}}{\sqrt{-i \pi y}}\left[2 \hat{\omega}_{\eta^{* e}} \mathcal{F}_{3 / 4}^{3 / 2}\left(\frac{\widetilde{\omega}_{D t}}{2}\right)\right. \\
& \left.\left.-\frac{3 \eta_{e} \widetilde{\omega}_{* e}}{2} \mathcal{F}_{7 / 4}^{3 / 2}\left(\frac{\widetilde{\omega}_{D t}}{2}\right)\right]\right\},
\end{aligned}
$$

where $\widetilde{\phi}=\left(1+4 i f_{s} / 5\right) 4 \phi_{0} /(3 \pi \phi), \quad \widetilde{\omega}_{D t}=\omega_{D 0} /\left(\omega x_{e}^{2}\right), \hat{\nu}_{t}=\hat{\nu} x_{e}^{3}$, $\widetilde{\omega}_{* a}=\omega_{* a} / \omega, \quad \hat{\omega}_{\eta_{* a}}=1-\left(1-3 \eta_{a} / 2\right) \widetilde{\omega}_{* a}, \quad$ and $\quad \mathcal{F}_{b}^{a}(z)={ }_{2} F_{0}$ $(a, b ; ; z)$, where ${ }_{2} F_{0}$ denotes the generalized hypergeometric function. To obtain Eq. (12), no approximation on the smallness of the normalized magnetic drift has been made and the hypergeometric functions incorporate the full effect of the drift resonances. The effect of the impurities is contained in the factor $1+Z_{\text {eff }}$ multiplying the collisionality parameter. Furthermore, the unstable mode frequencies and growth rates are affected by the impurities in certain conditions, as will be shown.

The solution in Eq. (11) reproduces the numerical solution of the GK equation more accurately than the WentzelKramers-Brillouin-solution used in Refs. 20 and 21, espe- cially in the case of complex electrostatic potentials. However, comparing the resulting perturbed electron density response (12) with the corresponding expression in Eq. (9) of Ref. 20, we find that the difference is only the appearance of a factor $4 / \pi$ in $\widetilde{\phi}$.

\section{B. Background ion and impurity responses}

For the ions, we neglect the parallel compressibility by assuming $k_{\|} v_{T i} \ll \omega$. In this limit, Eq. (1) can be solved neglecting the parallel derivative and replacing the magnetic drift frequency $\omega_{D i}$ with its weighted flux-surface averaged value $\left\langle\omega_{D i}\right\rangle_{\phi}$, where $\langle X(\theta)\rangle_{\phi}=\int_{-\pi}^{\pi} X(\theta) \phi(\theta) d \theta / \int_{-\pi}^{\pi} \phi(\theta) d \theta$, and thus the perturbed ion density becomes

$$
\begin{aligned}
\frac{\hat{n}_{i}}{n_{i}} / \frac{e \phi}{T_{i}}= & -\widetilde{\omega}_{* i}+\left(\frac{3 \widetilde{\omega}_{D s i}}{2}-b_{i}\right) \\
& \times\left[\hat{\omega}_{\eta_{* i}}-\frac{5}{2}\left(\eta_{i} \widetilde{\omega}_{* i}-\widetilde{\omega}_{D s i} \hat{\omega}_{\eta_{* i}}\right) \mathcal{F}_{7 / 2}^{1}\left(\widetilde{\omega}_{D s i}\right)\right] .
\end{aligned}
$$

Here, $\quad b_{a}=\left\langle b_{s a}\right\rangle_{\phi}=b_{a 0}\left\{1+s^{2}\left[2 \pi^{2}-12+i f_{s}\left(2 \pi^{2}-3\right)\right] /[6(1\right.$ $\left.\left.\left.+i f_{s}\right)\right]\right\}$ is the weighted flux-surface averaged value of the finite Larmor radius (FLR) parameter, $b_{s a}=b_{a 0}\left(1+s^{2} \theta^{2}\right)$, $b_{a 0}=\left(k_{\theta} \rho_{s a}\right)^{2}$, and $\rho_{s a}=v_{T a} / \sqrt{2} \omega_{c a}$. The averaged normalized magnetic drift frequency is

$$
\widetilde{\omega}_{D s a}=\frac{6+\left(9+16 i f_{s}\right) s}{12\left(1+i f_{s}\right)} \frac{\omega_{D a 0}}{\omega},
$$

where $\omega_{D a 0}=-2 k_{\theta} v_{T a}^{2} / 3 \omega_{c a} R$, and we used the constant energy resonance approximation for the ion resonance $\left[v_{\perp}^{2}\right.$ $\left.+2 v_{\|}^{2} \rightarrow 4\left(v_{\perp}^{2}+v_{\|}\right)^{2} / 3\right]{ }^{23}$ To obtain the expression for the perturbed ion response (13), only long-wavelength perturbations were considered, and only the terms linear in $b_{i 0}$ were kept. This approximation is typically valid for the fastest growing ion temperature gradient (ITG) modes $\left(k_{\theta} \rho_{s i} \sim 0.2\right)$.

For the impurities, collisions can be neglected as well, and thus the structure of the impurity solution is the same as for the ions and reads

$$
\begin{aligned}
\frac{\hat{n}_{z}}{n_{z}} / \frac{Z e \phi}{T_{z}}= & -\widetilde{\omega}_{* z}+\left(\frac{3 \widetilde{\omega}_{D s z}}{2}-b_{z}\right) \\
& \times\left[\hat{\omega}_{\eta^{* z}}-\frac{5}{2}\left(\eta_{z} \widetilde{\omega}_{* z}-\widetilde{\omega}_{D s z} \hat{\omega}_{\eta^{* z}}\right) \mathcal{F}_{7 / 2}^{1}\left(\widetilde{\omega}_{D s z}\right)\right] .
\end{aligned}
$$

\section{STABILITY}

The dispersion relation follows from the quasineutrality condition

$$
\frac{\hat{n}_{e}}{n_{e}}=\left(1-Z f_{z}\right) \frac{\hat{n}_{i}}{n_{i}}+Z f_{z} \frac{\hat{n}_{z}}{n_{z}},
$$

where $f_{z}=n_{z} / n_{e}$ is the fraction of impurities, and the perturbed electron, ion, and impurity densities are given by Eqs. (12), (13), and (14), respectively. The dispersion relation obtained here is valid for both ITG modes propagating in the ion diamagnetic direction $(\sigma=-1)$ and trapped electron modes propagating in the electron diamagnetic direction 

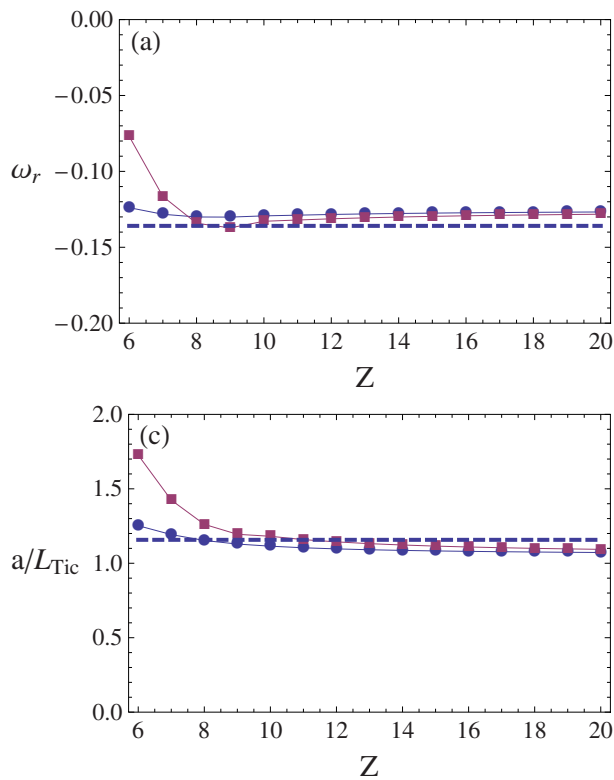
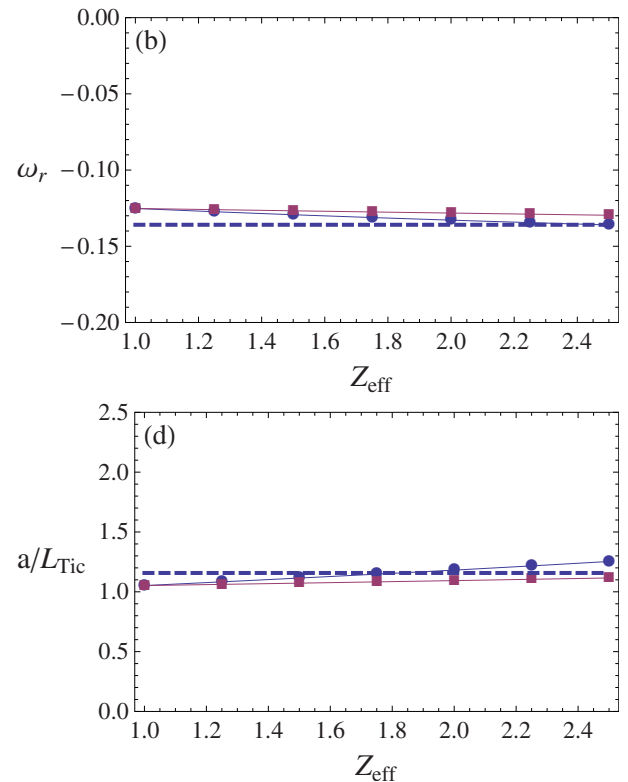

FIG. 1. (Color online) (a), (b) normalized mode frequency (in units of $c_{s} / a$ ) vs $Z$ (a) and $Z_{\text {eff }}$ (b). (c), (d) critical ion temperature gradient vs $Z$ (c) and $Z_{\text {eff }}$ (d). Dashed: analytical expression from Eqs. (17) and (18). (a), (c) dots: $Z_{\text {eff }}=1.5$; squares: $Z_{\text {eff }}=2$. (b), (d) dots: $Z=10$; squares: $Z=20$. The impurity charge or density does not affect the mode frequency or the critical ion temperature gradient significantly for $Z>10$.

$(\sigma=1)$, but in this paper we will focus only on the ITG mode stability and the quasilinear fluxes driven by them. In the limit of large aspect ratio, $\epsilon \rightarrow 0$, the trapped part of the perturbed electron density can be neglected, and the dispersion relation for ITG $(\sigma=-1)$ modes with adiabatic electrons reduces to the following expression:

$$
\begin{aligned}
1= & \tau_{i}\left(Z f_{z}-1\right)\left\{\widetilde{\omega}_{* i}-\left(\frac{3 \widetilde{\omega}_{D s i}}{2}-b\right)\right. \\
& \left.\times\left[\hat{\omega}_{\eta^{* i}}-\frac{5}{2}\left(\eta_{i} \widetilde{\omega}_{* i}-\widetilde{\omega}_{D s i} \hat{\omega}_{\eta * i}\right) \mathcal{F}_{7 / 2}^{1}\left(\widetilde{\omega}_{D s i}\right)\right]\right\} \\
& -Z^{2} f_{z} \tau_{z}\left\{\widetilde{\omega}_{* z}-\left(\frac{3 \widetilde{\omega}_{D s z}}{2}-b_{z}\right)\right. \\
& \left.\times\left[\hat{\omega}_{\eta^{* z}}-\frac{5}{2}\left(\eta_{z} \widetilde{\omega}_{* z}-\widetilde{\omega}_{D s z} \hat{\omega}_{\eta^{* z}}\right) \mathcal{F}_{7 / 2}^{1}\left(\widetilde{\omega}_{D s z}\right)\right]\right\},
\end{aligned}
$$

where $\tau_{a}=T_{e} / T_{a}$. Using the condition for marginal stability, $\gamma=0$, we can derive an approximate stability condition for the ITG modes. As in Ref. 20, we note that if the imaginary parts of $\widetilde{\omega}_{D s a}$ and $b_{a}$ are negligible (which is the case if $f_{s} \ll 1$ at marginal stability $\gamma=0$ ), then the expression in Eq. (16) is real except for the terms containing the functions $\mathcal{F}_{7 / 2}^{1}\left(\widetilde{\omega}_{D s i}\right)$ and $\mathcal{F}_{7 / 2}^{1}\left(\widetilde{\omega}_{D s z}\right)$ that have imaginary parts for all values except $\widetilde{\omega}_{D s i}=0$ and $\widetilde{\omega}_{D s z}=0$. We also note that for impurity charge numbers $Z \gtrsim 10$, the imaginary part of the impurity hypergeometric function is negligible compared to the ion term. Therefore, in this limit, the condition $\gamma=0$ can only be satisfied if the coefficient in front of $\mathcal{F}_{7 / 2}^{1}\left(\widetilde{\omega}_{D s i}\right)$ vanishes. This leads to the conclusion that for higher impurity charge numbers, the eigenfrequency and stability boundary are very weakly affected by the increasing impurity charge, even for a significant fraction of impurities, and are approximately equal to the corresponding quantities in a pure plasma, ${ }^{20}$

$$
\begin{aligned}
& \frac{\omega_{0 c}}{\omega_{* e}}=\frac{b-1}{\tau_{i} b+1}+\left(1+\frac{1}{\tau_{i}}\right) \frac{(2+3 s) L_{n i}}{\left(\tau_{i} b+1\right) 2 R}, \\
& \frac{a}{L_{\text {Tic }}}=\frac{\left(1+\tau_{i}^{-1}\right)(2+3 s) a}{3 R(1-b)} .
\end{aligned}
$$

In Fig. 1, we show the mode frequency $\omega_{0}$ (normalized to $c_{s} / a$, where $c_{s}=\sqrt{T_{e} / m_{i}}$ is the ion sound speed) and critical temperature gradient $a / L_{\text {Tic }}$ computed from the full dispersion relation (including the nonadiabatic electrons) as a function of the impurity charge $Z$ and $Z_{\text {eff }}$, together with the expressions given in Eqs. (17) and (18). The parameters used in the calculations were $s=1, q=2, a / R=1 / 3, a / r=2, a / L_{n e}$ $=1$, and $k_{\theta} \rho_{s}=0.2$. The agreement with the analytical expressions is excellent, except for impurities with lower charge numbers, where the impurity hypergeometric function is of the same order of magnitude as that of the ions. For lower impurity charge, especially for helium or carbon dilutions, impurities are expected to significantly influence the eigenfrequency and thus the stability boundary if the amount of impurities is not too small.

\section{QUASILINEAR PARTICLE FLUXES}

The quasilinear particle flux for species $a$ is given by

$$
\Gamma_{a}=-\frac{k_{\theta} p_{a}}{e B}\left|\frac{e_{a} \bar{\phi}}{T_{a}}\right|^{2} \mathfrak{I}\left(\frac{\hat{n}_{a} / n_{a}}{e_{a} \bar{\phi} / T_{a}}\right),
$$

where the bar denotes flux-surface averaged quantities, $\bar{\phi}$ $=\left(1+i f_{s}\right) \phi_{0} / 2$, and $\hat{n}_{a} / n_{a}$ is the nonadiabatic perturbed density response. The quasilinear fluxes are evaluated using the expressions for the perturbed electron, ion and impurity densities from Eqs. (12)-(14), respectively, and solving the 

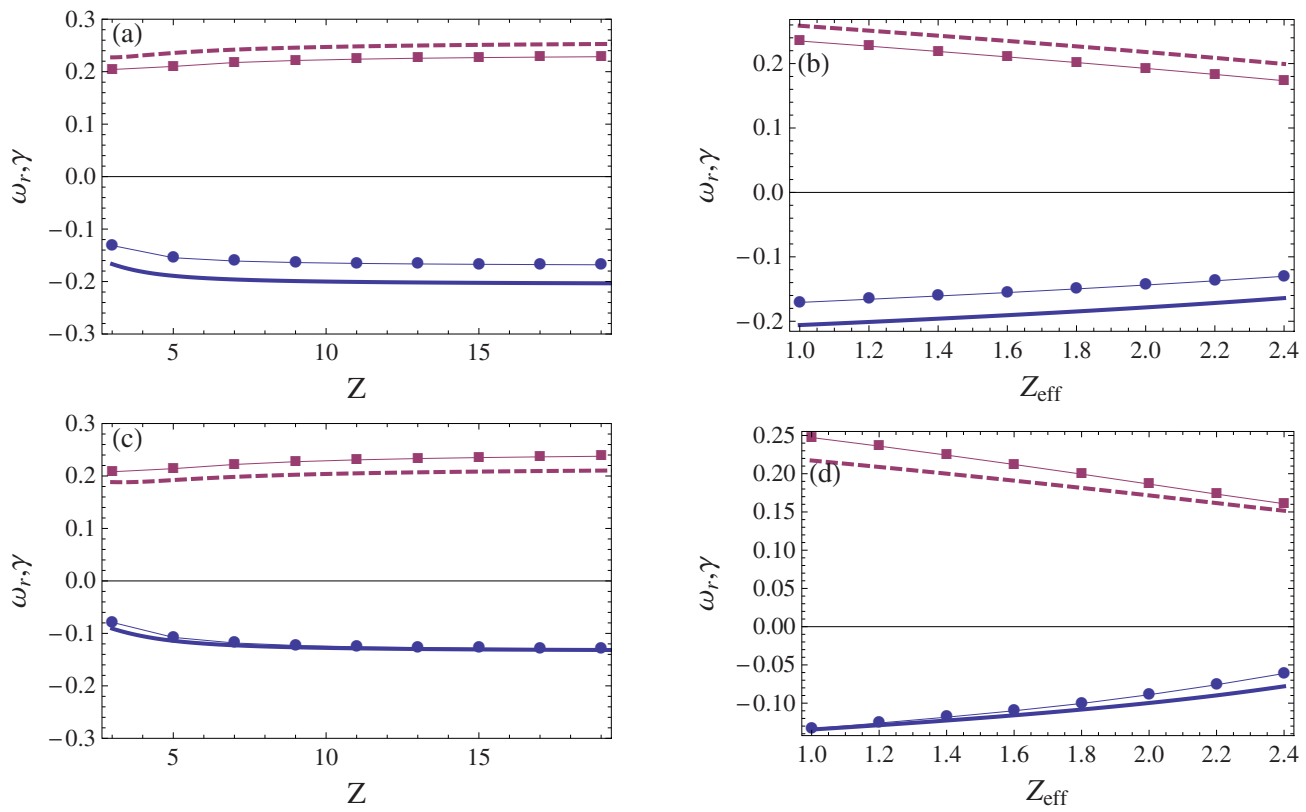

FIG. 2. (Color online) (a), (c) normalized mode frequency (solid) and growth rate (dashed) (in units of $c_{s} / a$ ) vs $Z$ for $Z_{\text {eff }}=1.5$. (b), (d) mode frequency (solid) and growth rate (dashed) vs $Z_{\text {eff }}$ for $Z=6$. The dots and squares are the corresponding GYRO results. (a), (b) GA standard case. (c), (d) hybrid case.

dispersion relation (15), including the nonadiabatic electron response, for the unstable mode frequencies and growth rates. In the following, we will present scalings of the eigenfrequency, growth rate, and impurity particle fluxes with impurity charge $Z$, effective charge $Z_{\text {eff }}$, inverse impurity density scale length $a / L_{n z}$, and collisionality, together with quasilinear GYRO results for the GA standard case, ${ }^{22} a / L_{T e}$ $=a / L_{T i}=3, s=1, q=2, a / R=1 / 3, r / a=1 / 2, a / L_{n e}=1$, and $k_{\theta} \rho_{s}=0.2$, and parameters relevant to the inner core of hybrid plasmas, $a / L_{T e}=a / L_{T i}=3, \quad s=1, \quad q=1.25, a / R=1 / 3, \quad r / a$ $=1 / 4, a / L_{n e}=1.5$, and $k_{\theta} \rho_{s}=0.2$. All the frequencies and growth rates are given in units of $c_{s} / a$, and the fluxes are normalized to $k_{\theta} p_{e} / e B\left|e \bar{\phi} / T_{e}\right|^{2}$.

\section{A. $Z$ - and $Z_{\text {eff }}$-scalings}

For moderate $Z$ (i.e., $10 \leqq Z \leqq 25$ ), the eigenfrequency and growth rate of the unstable modes are only weakly affected by increasing $Z$. The main reason for this is the fact that the imaginary part of the impurity hypergeometric function is negligible compared to the ion term for $Z \gtrsim 10$. The absolute values of the eigenfrequencies and growth rates decrease slightly with increasing $Z_{\text {eff }}$, reflecting the fact that the impurity terms of the dispersion relation start to play a larger role when the impurity density is increased, and the presence of impurities is stabilizing. In Fig. 2, we show the scalings with $Z$ and $Z_{\text {eff }}$ for the GA standard and hybrid cases, together with the results of quasilinear GYRO simulations.

Figure 3 shows that the normalized impurity flux is expected to be outward for the cases we studied. As we will show later, for these parameters, this is expected when $R / L_{n z} \gtrsim 2$. The rapid fall in Fig. 3(a) with increasing $Z$ is due to the fact that we keep $Z_{\text {eff }}$ constant. Although the impurity flux is not sensitive to $Z$, the normalized impurity flux is rapidly decreasing since it is proportional to $n_{z} / n_{e} \sim 1 / Z^{2}$. The normalized flux increases for increasing impurity den- sity and this is more pronounced for impurities with lower charge numbers since the relative increase in $Z_{\text {eff }}$ is larger than for high charge numbers. The ion and electron fluxes are expected to be inward and their absolute values decrease with increasing $Z$.

\section{B. $a / L_{n z}$-scaling}

The eigenfrequencies and growth rates depend moderately on the inverse impurity density gradient, and the particle fluxes show a strong dependence, as illustrated in Fig. 4, for the GA standard and hybrid cases. The impurity flux changes sign at a certain fixed value of the radial impurity density gradient. For the GA standard case with $Z_{\text {eff }}=1.5$ shown in Fig. 4(b), the crossovers are at $a / L_{n z} \simeq 0.59$ for $Z=6$ and $a / L_{n z} \simeq 0.68$ for $Z=10$, and for the hybrid case shown in Fig. 4(d) it is at $a / L_{n z} \simeq 0.62$ for $Z=6$ and $a / L_{n z}$ $\simeq 0.67$ for $Z=10$. For $Z_{\text {eff }}=2$ and $Z=6$, the crossover point shifts to $a / L_{n z} \simeq 0.55$ for the GA standard case and $a / L_{n z}$ $\simeq 0.58$ for the hybrid case. Thus, the crossover point is almost at the same $a / L_{n z}$ independently of the charge number $Z$, effective impurity charge $Z_{\text {eff }}$, and inverse background temperature scale lengths. Using the perturbed impurity density from Eq. (14), we can derive an approximation for the crossover point by noting that the impurity hypergeometric function can be replaced by its asymptotic limit for small arguments, $\mathcal{F}_{7 / 2}^{1}\left(\widetilde{\omega}_{D s z}\right)=1$ (which is a good approximation for heavy impurities since the argument $\widetilde{\omega}_{D s z} \propto 1 / Z$ ). If we also assume that the impurity FLR-term is negligible, $b_{z}$ $\ll 3 \widetilde{\omega}_{D s z} / 2$, the crossover point is expected to be at

$$
\Im \widetilde{\omega}_{* z}=\Im\left\{\frac{3 \widetilde{\omega}_{D s z}}{2}\left[\hat{\omega}_{\eta^{* z}}-\frac{5}{2}\left(\eta_{z} \widetilde{\omega}_{* z}-\widetilde{\omega}_{D s z} \hat{\omega}_{\eta^{* z}}\right)\right]\right\} .
$$

In the limit of moderate or high impurity charge, all terms of 

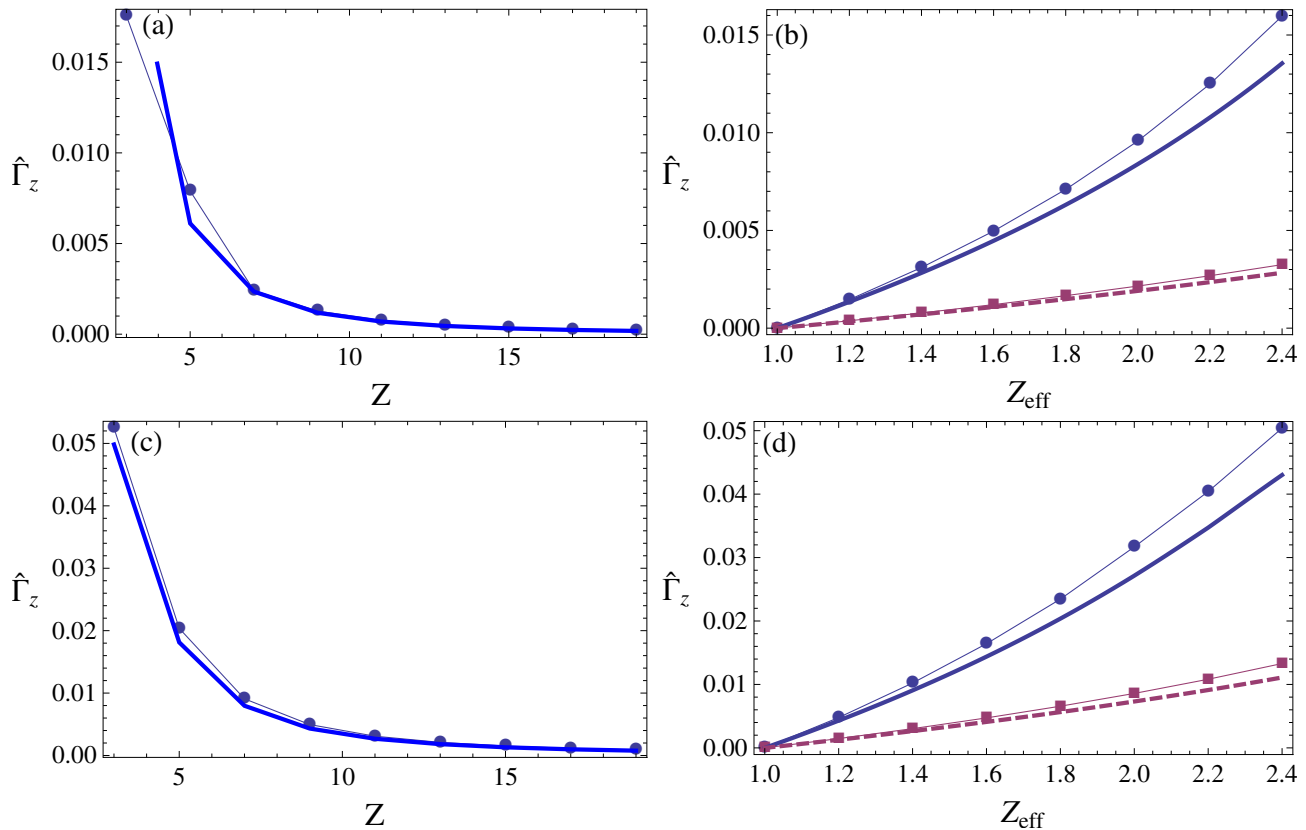

FIG. 3. (Color online) (a), (c) normalized impurity flux vs impurity charge $Z$ for $Z_{\text {eff }}=1.5$. (b), (d) normalized impurity flux vs $Z_{\text {eff }}$ for $Z=6$ (solid) and $Z$ $=10$ (dashed). The dots and squares are the corresponding GYRO results. (a), (b) GA standard case. (c), (d) hybrid case.

order $1 / Z^{2}$ can be neglected so Eq. (20) simplifies to $\Im \widetilde{\omega}_{* z}=\Im\left\{3 \widetilde{\omega}_{D s z} / 2\right\}$. Using the approximation $\widetilde{\omega}_{D s z}$ $\simeq(2+3 s) \omega_{D i 0} / 6 \omega$ (valid if $\left.f_{s} \ll 1\right)$, the zero-flux impurity density gradient becomes $R / L_{n z c}=(2+3 s) / 2$. This is somewhat larger than the value obtained keeping all the terms in the expression for the impurity flux (which for the GA standard case and $Z=10$ is $R / L_{n z c} \simeq 2$ ). Note that to arrive at Eq. (20), we only assumed high $Z$ and $b_{z} \ll 3 \widetilde{\omega}_{D s z} / 2$, no assumptions on the eigenfrequency or the magnitude of other parameters have been used. It is interesting to note that for high $Z$ the zero-flux impurity density gradient becomes nearly independent of the charge number. Previously, similar results have been obtained in fluid simulations of ITG turbulence dominated transport. ${ }^{5}$ The physical reason for this is that the convective flux originating from the curvature drift is nearly independent of the charge number, while the convection caused by thermodiffusion decreases with increasing $Z$ (this corresponds to the term proportional to $\eta_{z}$ ).

For moderate and high $Z$, the expression for the zero-flux impurity density gradient can be refined to be
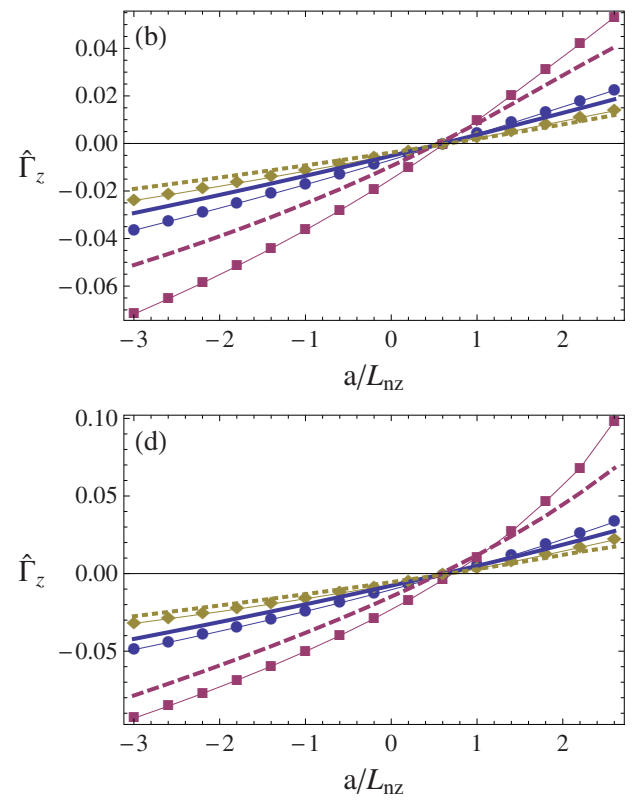

FIG. 4. (Color online) (a), (c) normalized mode frequency (solid) and growth rate (dashed) (in units of $c_{s} / a$ ) vs inverse radial impurity density gradient for $Z=6$ and $Z_{\text {eff }}=1.5$. (b), (d) normalized impurity particle flux vs inverse radial impurity density gradient for the parameters: solid: $Z_{\text {eff }}=1.5, Z=6$, dashed: $Z_{\text {eff }}=2, Z=6$, and dotted: $Z_{\text {eff }}=2, Z=10$. The dots and squares are the corresponding GYRO results. (a), (b) GA standard case. (c), (d) hybrid case. 

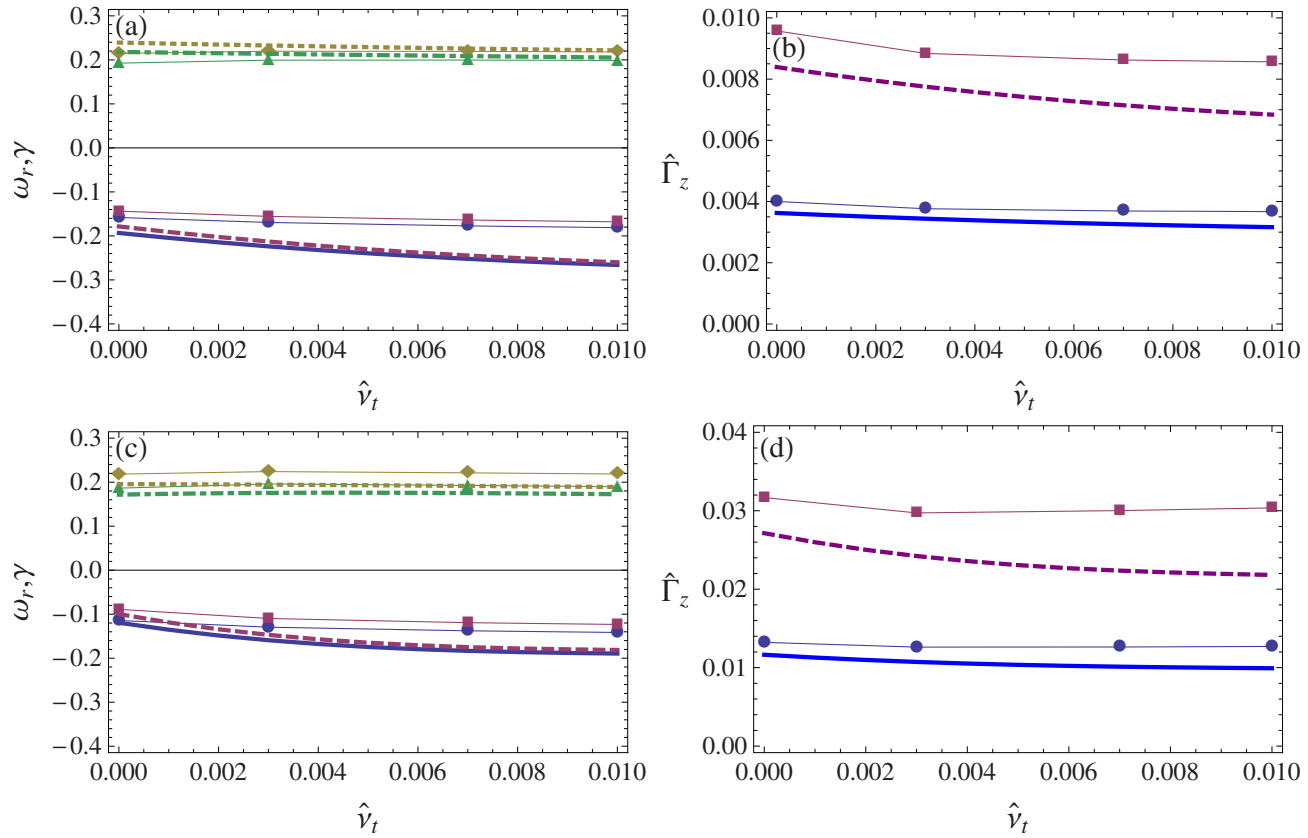

FIG. 5. (Color online) Normalized mode frequency, growth rate [(a) and (c)] and normalized impurity particle flux [(b) and (d)] vs normalized collisionality (in $c_{s} / a$ ) for $Z=6$ and (solid) $Z_{\text {eff }}=1.5$ and (dashed) $Z_{\text {eff }}=2$. The dots and squares are the corresponding GYRO results. (a), (b) GA standard case. (c), (d) hybrid case.

$$
\frac{R}{L_{n z c}}=\frac{(2+3 s)}{2} \frac{1-\frac{2}{1+\hat{\gamma}^{2}} \frac{k_{\theta} \rho_{s}}{Z \tau_{z} \omega_{0}^{n}}\left(\frac{a}{L_{T z}}-\frac{a}{R} \frac{(2+3 s) 5}{6}\right)}{1+\frac{a}{R} \frac{2+3 s}{1+\hat{\gamma}^{2}} \frac{k_{\theta} \rho_{s}}{Z \tau_{z} \omega_{0}^{n}}},
$$

where we neglected terms of order $1 / Z^{3}$ in Eq. (20). Here, $\omega_{0}^{n}$ is $\omega_{0}$ normalized to $c_{s} / a$. For the GA standard case, this expression gives $a / L_{n z c}=0.58$ for $Z=6$ and $a / L_{n z c}=0.67$ for $Z=10$, which are in excellent agreement with the values that are obtained by keeping all the terms in the expression for the impurity flux (here we used $\hat{\gamma}=1.2$ and $\omega_{0}^{n}=0.2$ ). Equation (21) shows that for higher impurity temperature gradient or higher $k_{\theta} \rho_{s}$ the zero-flux impurity density gradient is lower, a trend which is in good agreement with our numerical results.

In the approximate analysis above we assumed the unstable mode frequencies and growth rates to be constant, as, for the same set of parameters, they do not show a strong dependence on the charge number or impurity density. However, if, for instance, the inverse electron density scale length $a / L_{n e}$ or the temperature ratio $\tau_{i}$ are changed, the unstable mode frequencies and growth rates will also change and the zero-flux impurity density gradient will be affected by that, especially for low $Z$ when the effect of thermodiffusion cannot be neglected. This means that in scenarios with more peaked electron density profiles or strongly differing electron-to-ion temperature ratios, the zero-flux impurity density gradient is expected to be different from that in scenarios with flat density profiles or if $\tau_{i}=1$. An indication of this can be seen by comparing the hybrid case with $a / L_{n e}$ $=1.5$ with the GA standard case with $a / L_{n e}=1$. The hybrid case leads to a higher normalized growth rate, and, due to the terms proportional to $1 /\left(1+\hat{\gamma}^{2}\right)$ in the expression for $R / L_{n z c}$, this leads to a slightly larger $R / L_{n z c}$ for $Z=6$. $R / L_{n z c}$ for $Z=10$ is the same in these two cases because for higher $Z$, the effect of the terms proportional to $1 /\left(1+\hat{\gamma}^{2}\right)$ is reduced.

\section{Collisionality scaling}

Previous work noted that collisions do not affect the impurity fluxes significantly. ${ }^{3}$ The results of the GYIMES model confirm this. In Fig. 5, it is shown that the unstable mode frequencies are slightly decreasing with increasing collisionality but the growth rates are almost independent of collisionality in both the GA standard and hybrid cases. The effect of collisions on the impurity flux only enters through the eigenfrequency, which means that the impurity flux is only very weakly dependent on collisionality. This is in contrast to the very sensitive dependence of the electron particle flux on collisionality, for which, in general, a sign change from inward to outward is expected at very small collisionalities. $^{1,2,20,21,25}$

\section{CONCLUSIONS}

In this paper we presented a semianalytical model for impurity transport driven by electrostatic turbulence. The model does not rely on expansions in the smallness of the magnetic drift frequencies, and includes collisions modeled by a Lorentz operator. By assuming large aspect ratio, low beta, toroidal symmetry, circular cross section, and weak collisionality, and assuming a ballooning eigenfunction for the electrostatic potential, analytical expressions can be derived for the ion, impurity, and electron perturbed densities and the quasilinear fluxes. The semianalytical character of the model eases the interpretation of experimental and simulation re- 

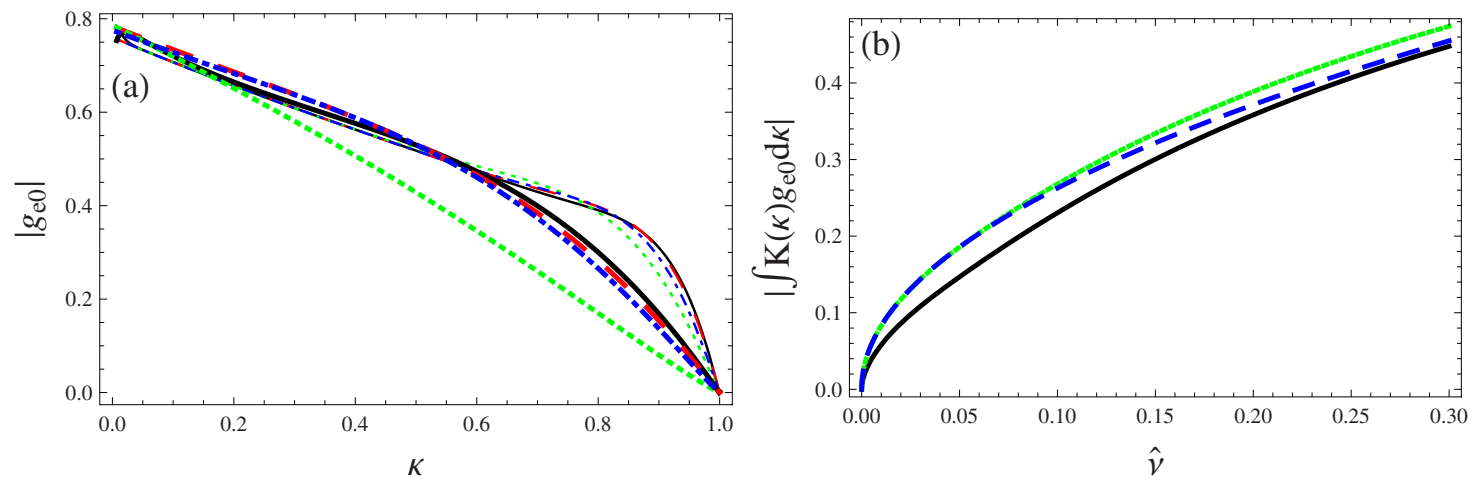

FIG. 6. (Color online) (a) Absolute value of the trapped electron distribution function as a function of the trapping parameter $\kappa$ for $f_{s}=0.18, y=-1+0.2 i$, and $\widetilde{\omega}_{D}=0.6$. Solid (black) line is the numerical solution of Eq. (5), dashed (red) line is the boundary layer solution from Eq. (11), dotted line is Eq. (11) using the asymptotic value of the elliptic integral $K(\kappa)$ for $\kappa=1(K(\kappa) \simeq \ln \hat{\nu} / 4)$ in the exponent, and dot-dashed (blue) line is Eq. (11) using a constant approximation for $K(\kappa)=2$ in the exponent. Thin lines are for $\hat{\nu}=0.01$ and thick lines are for $\hat{\nu}=0.1$. The dot-dashed line approximates the full numerical solution very well for all collisionalities and this is used in the calculations. (b) Absolute value of the collisional part of the $\kappa$-integral of the perturbed electron distribution function: (solid) $\int_{0}^{1} K(\kappa)\langle\phi\rangle \exp [(\kappa-1) \sqrt{K(\kappa) /(2 n)}] d \kappa ;$ (dotted) $4 / 3\left[1+\left(i 4 f_{s} / 5\right)\right] \int_{0}^{1} \exp [(\kappa-1) / \sqrt{n}] d \kappa$; (dashed) $(4 / 3)\left[1+\left(i 4 f_{s} / 5\right)\right] \sqrt{n}$. The parameters are the same as in (a).

sults. In this paper, we study only ITG turbulence dominated cases, but the model is suitable for trapped electron mode turbulence as well.

For moderate or high impurity charge number $Z>10$, the eigenfrequency and stability boundary are very weakly affected by the increasing impurity charge for constant effective charge, even for a significant fraction of impurities in the plasma, and are approximately equal to the corresponding quantities in a pure plasma. For lower impurity charge, the effect of the impurities influences the eigenfrequency and thus the stability boundary significantly if the amount of impurities is not too small.

If the mode is far from marginal stability, the effect of increasing charge number and density affects the growth rates and mode frequencies only weakly. Furthermore, the impurity particle flux is only very weakly dependent on the charge number. The normalized impurity flux is reduced with increasing charge number if $Z_{\text {eff }}$ is kept constant, but that is an artifact of the reducing impurity fraction $n_{z} / n_{e} \sim 1 / Z^{2}$. The impurity flux changes sign from inward to outward at approximately the same value of $a / L_{n z}$ independently of $Z$, $Z_{\text {eff }}$, and many other plasma parameters. This has been noted previously, ${ }^{5}$ but here we also derive an approximate analytical expression for the zero-flux impurity density gradient $R / L_{n z c}$ using the analytical expression for the perturbed impurity density. This is useful to gain better understanding of the parametric dependencies of $R / L_{n z c}$. We find that collisions do not affect the mode frequencies, growth rates, and impurity fluxes significantly, in agreement with previous results. $^{3}$

The results of the impurity transport model presented in this paper agree well with quasilinear GK simulations with GYRO. It includes some effects that previously have been neglected in analytical calculations, for instance, collisions and magnetic drifts are treated with more accuracy than in other existing semianalytical models, but it is still simple enough to ease the interpretation of certain physical effects, as we illustrated with the approximation for the zero-flux impurity density gradient. Due to its simplicity, it is straight- forward to extend it by including several impurity species or include it in transport simulations. However, due to the model electrostatic potential used in the calculations, reliable quantitative predictions can only be obtained in the moderate shear region.

\section{ACKNOWLEDGMENTS}

This work was funded by the European Communities under Association Contract between EURATOM, Vetenskapsrådet and Germany. The views and opinions expressed herein do not necessarily reflect those of the European Commission. The authors would like to thank J. Candy for providing the GYRO code and $\mathrm{H}$. Nordman and P. Helander for fruitful discussions.

\section{APPENDIX: DERIVATION OF THE PERTURBED ELECTRON DENSITY RESPONSE}

The perturbed electron density response is proportional to

$$
\left\langle\int g_{e 0} d^{3} v\right\rangle=4 \sqrt{2 \epsilon} \int_{0}^{\infty} v^{2} d v \int_{0}^{1} K(\kappa) g_{e 0} d \kappa,
$$

where, using the solution from Eq. (11), $g_{e 0}=g_{\text {outer }}(1$ $-\exp [-(1-\kappa) \sqrt{\hat{u} K(\kappa) / \hat{\nu}}])$ and the identity

$$
\begin{aligned}
& \int_{0}^{1}\left\{E(\kappa)+i \frac{4 f_{s}}{3}[(2 \kappa-1) E(\kappa)+(1-\kappa) K(\kappa)]\right\} d \kappa \\
& =\frac{4}{3}\left(1+i \frac{4 f_{s}}{5}\right),
\end{aligned}
$$

the $\kappa$-integral can be evaluated. To make analytical progress we will approximate the elliptic integral in the exponent of $g_{e 0}$ with a constant value $K(\kappa) \simeq \int_{0}^{1} K(\kappa) d \kappa=2$. To show the validity of this approximation Fig. 6(a) compares the absolute value of $g_{e 0}$ as a function of the trapping parameter $\kappa$ from a numerical solution of Eq. (5), and the boundary layer solution from Eq. (11) for two collisionalities $(\hat{\nu}=0.01$ and 
$\hat{\nu}=0.1)$. We show also with dotted line the solution from Eq. (11) using the asymptotic value of the elliptic integral $K(\kappa)$ for $\kappa=1(K(\kappa) \simeq \ln \hat{\nu} / 4)$ in the exponent, and the dotdashed line is Eq. (11) using a constant approximation for $K(\kappa)=2$ in the exponent. The dot-dashed line approximates the full numerical solution very well for all collisionalities and this is used in our calculations. Interestingly, the approximation using the asymptotic value of the elliptic integral breaks down for large collisionalities. The reason for this is that the logarithmic behavior of the elliptic integral is only exhibited very close to $\kappa=1$, and therefore, for larger (experimentally relevant) collisionalities, when the width of the boundary layer is larger, the logarithmic value is not a good approximation to the elliptic integral. Using the approximation $\left\langle\omega_{D e}\right\rangle \simeq \omega_{D 0} / 2$ and $g_{e 0}=g_{\text {outer }} \quad(1-\exp [-(1$ $-\kappa) \sqrt{2 \hat{u} / \hat{\nu}}])$ the $\kappa$-integral becomes

$$
\begin{aligned}
\int_{0}^{1} K(\kappa) g_{e 0} d \kappa \simeq & \left.-\frac{4 e \phi_{0} f_{e 0}\left(\frac{\omega-\omega_{* e}^{T}}{3 T_{e}}\right)\left(1+i \frac{4 f_{s}}{5}\right)}{\omega-\omega_{D 0} / 2}\right) \\
& \times\left(1-\sqrt{\frac{i \hat{\nu}}{y\left(2-\widetilde{\omega}_{D}\right)}}\right),
\end{aligned}
$$

where $\widetilde{\omega}_{D}=\omega_{D 0} / \omega$ denotes the normalized magnetic drift frequency. To obtain an analytical expression for the collisional part of the integral in Eq. (A2), we approximated $\int_{0}^{1} K(\kappa)$ $\times\langle\phi\rangle \exp [(\kappa-1) / \sqrt{n}] d \kappa \simeq \int_{0}^{1} K(\kappa)\langle\phi\rangle d \kappa \int_{0}^{1} \exp [(\kappa-1) / \sqrt{n}] d \kappa$ $=4 / 3\left(1+i 4 f_{s} / 5\right) \sqrt{n}$, with $n=i \hat{v} / y\left(2-\widetilde{\omega}_{D}\right)$, and after integration retained terms only to the lowest order in $\hat{\nu}^{1 / 2}$. A comparison with the numerical solution confirms that the approximate expression (A2) agrees very well with the correct value. In Fig. 6(b), we illustrate the good agreement between the full integral and its approximation by showing with solid line the numerical solution of the collisional part of the $\kappa$-integral from Eq. (A1), including the $\kappa$-variation in the averaged electrostatic potential $\int_{0}^{1} K(\kappa)\langle\phi\rangle \exp [(\kappa$ $-1) \sqrt{K(\kappa) /(2 n)}] d \kappa$, with dotted we show $4 / 3(1$ $\left.+i 4 f_{s} / 5\right) \int_{0}^{1} \exp [(\kappa-1) / \sqrt{n}] d \kappa$, and with dashed line $4 / 3(1$ $\left.+i 4 f_{s} / 5\right) \sqrt{n}$. The latter is the approximation we use to obtain the expression in Eq. (A2).

${ }^{1}$ C. Estrada-Mila, J. Candy, and R. E. Waltz, Phys. Plasmas 12, 022305 (2005).

${ }^{2}$ C. Angioni and A. G. Peeters, Phys. Rev. Lett. 96, 095003 (2006).

${ }^{3}$ C. Angioni, A. G. Peeters, G. V. Pereverzev, A. Bottino, J. Candy, R. Dux,

E. Fable, T. Hein, and R. E. Waltz, Nucl. Fusion 49, 055013 (2009).

${ }^{4}$ T. Hein and C. Angioni, Phys. Plasmas 17, 012307 (2010).

${ }^{5}$ T. Fülöp and H. Nordman, Phys. Plasmas 16, 032306 (2009).

${ }^{6}$ H. Nordman, R. Singh, T. Fülöp, L.-G. Eriksson, R. Dumont, J. Anderson, P. Kaw, P. Strand, M. Tokar, and J. Weiland, Phys. Plasmas 15, 042316 (2008).

${ }^{7}$ H. Nordman, T. Fülöp, J. Candy, P. Strand, and J. Weiland, Phys. Plasmas 14, 052303 (2007).

${ }^{8}$ N. Dubuit, X. Garbet, T. Parisot, R. Guirlet, and C. Bourdelle, Phys. Plasmas 14, 042301 (2007).

${ }^{9}$ R. Guirlet, C. Giroud, T. Parisot, M. E. Puiatti, C. Bourdelle, L. Carraro, N. Dubuit, X. Garbet, and P. R. Thomas, Plasma Phys. Controlled Fusion 48, B63 (2006).

${ }^{10}$ T. Fülöp and J. Weiland, Phys. Plasmas 13, 112504 (2006).

${ }^{11}$ M. Fröjdh, M. Liljeström, and H. Nordman, Nucl. Fusion 32, 419 (1992).

${ }^{12}$ C. Bourdelle, X. Garbet, G. T. Hoang, J. Ongena, and R. V. Budny, Nucl. Fusion 42, 892 (2002).

${ }^{13}$ C. Bourdelle, X. Garbet, F. Imbeaux, A. Casati, N. Dubuit, R. Guirlet, and T. Parisot, Phys. Plasmas 14, 112501 (2007).

${ }^{14}$ S. Moradi, M. Z. Tokar, R. Singh, and B. Weyssow, Nucl. Fusion 49, 085007 (2009).

${ }^{15}$ S. Moradi, M. Z. Tokar, and B. Weyssow, Phys. Plasmas 17, 012101 (2010).

${ }^{16}$ E. A. Belli and J. Candy, Plasma Phys. Controlled Fusion 50, 095010 (2008).

${ }^{17}$ S. P. Hirshman and D. J. Sigmar, Nucl. Fusion 21, 1079 (1981).

${ }^{18} \mathrm{P}$. Helander and D. J. Sigmar, Collisional Transport in Magnetized Plasmas (Cambridge University Press, Cambridge, 2002).

${ }^{19}$ A. Casati, C. Bourdelle, X. Garbet, F. Imbeaux, J. Candy, F. Clairet, G. Dif-Pradalier, G. Falchetto, T. Gerbaud, V. Grandgirard, Ö. D. Gürcan, P. Hennequin, J. Kinsey, M. Ottaviani, R. Sabot, Y. Sarazin, L. Vermare, and R. E. Waltz, Nucl. Fusion 49, 085012 (2009).

${ }^{20}$ I. Pusztai, T. Fülöp, J. Candy, and J. R. Hastie, Phys. Plasmas 16, 072305 (2009).

${ }^{21}$ T. Fülöp, I. Pusztai, and P. Helander, Phys. Plasmas 15, 072308 (2008).

${ }^{22}$ J. Candy and R. E. Waltz, J. Comput. Phys. 186, 545 (2003).

${ }^{23}$ F. Romanelli and S. Briguglio, Phys. Fluids B 2, 754 (1990).

${ }^{24}$ J. W. Connor, R. J. Hastie, and J. B. Taylor, Proc. R. Soc. London, Ser. A 365, 1 (1979).

${ }^{25}$ C. Angioni, A. G. Peeters, F. Jenko, and T. Dannert, Phys. Plasmas 12, $112310(2005)$ 